\title{
Detection of Mycobacterial Lipoarabinomannan with A Monoclonal Antibody Qualitative ELISA in Urine of Tuberculous Meningitis Patients
}

\author{
Sylvia Rachmayati ${ }^{1}$, Anita Liliana Susanti ${ }^{2, *}$, Basti Andriyoko ${ }^{1}$ \\ ${ }^{1}$ Clinical Pathology Department, Faculty of Medicine, Padjadjaran University, Jl. Eijkman No.38, Bandung, Indonesia \\ ${ }^{2}$ Clinical Pathology Department, Faculty of Medicine, General Achmad Yani University, Jl. Terusan Jenderal Sudirman, Cimahi, Indonesia \\ *Corresponding author. E-mail: anitalee.dr@gmail.com
}

Received date: May 19, 2015; Revised date: Dec 7, 2015; Accepted date: Jan 8, 2016

\section{Abstract}

$\mathrm{B}$ ACKGROUND: Tuberculous meningitis is the most severe manifestation of tuberculosis. The diagnostic approach of tuberculous meningitis is difficult. Combination of clinical, laboratory and radiological criteria were used in diagnostic approach of tuberculous meningitis. Urinary mycobacterial lipoarabinomannan (LAM) antigen detection is a promising diagnostic tool. Detection of mycobacterial antigen in concentrated urine sample is predicted to improve the positivity rate of the qualitative enzyme-linked immunosorbent assay (ELISA) diagnostic tool. The purpose of this study is to examine the detection ability of a monoclonal antibody qualitative ELISA in concentrated and unconcentrated urine of tuberculous meningitis patients.

METHODS: This research is a descriptive, crosssectionally designed. The study was conducted in the Clinical Pathology Department laboratory of Dr. Hasan Sadikin Hospital, in July-October 2014. A total of 27 patients diagnosed as tuberculous meningitis patients were included and the subjects were classified into possible and probable criteria according to consensus criteria. The subjects were classified as definite if the cerebrospinal fluid culture was positive for Mycobacterial tuberculosis growth. The subjects were examined for the presence of LAM in unconcentrated and concentrated urine with a monoclonal antibody qualitative ELISA method.

RESULTS: Unconcentrated urinary LAM examination positivity was $0 \%$ while in concentrated urine was $14.8 \%$. The positivity of concentrated urinary LAM were higher among the definite criteria group.

CONCLUSION: Concentrating urine sample increase the positivity rate of urinary LAM detection with ELISA method as high as $14.8 \%$. The urinary antigen detection is higher among the definite tuberculous meningitis patients.

KEYWORDS: LAM, concentrated urine, tuberculous meningitis, qualitative ELISA

Indones Biomed J. 2016; 8(1): 55-60

\section{Introduction}

Tuberculosis is one of major health problems in the world, the World Health Organization (WHO) in 1993 stated that tuberculosis is a global public health emergency.(1-3) In 2011, the burden of tuberculosis disease is highest in Asia and Africa. Indonesia is one country with large burden of tuberculosis (0.4-0.5 million). The incidence of mortality due to tuberculosis is $150-299$ deaths per 100,000 population in 2011.(1)

Tuberculosis can be manifested as pulmonary or extrapulmonary tuberculosis.(4) The most severe form of tuberculosis is tuberculous meningitis. Diagnosis of tuberculous meningitis is difficult and relied on history, physical examination, laboratory and radiological 
examination in a scoring system. Consensus scoring system is the most widely used scoring system to diagnose tuberculous meningitis. One of the important points in the diagnosis of tuberculous meningitis with consensus scoring system is the presence of Mycobacterial tuberculosis (M. tuberculosis) infection somewhere else other than in the Central Nervous System (CNS).(5) Diagnostic tests that may indicate the presence of $M$. tuberculosis infection, regardless of the anatomic location of the infection, can be very helpful in the diagnosis of tuberculous meningitis.

The cell wall of M. tuberculosis has a high lipid content and composed of a complex, which consists of mycolic acid, arabinogalactan and peptidoglycan (MAGP). MAGP complex is the largest component of mycobacterial wall. Lipoarabinomanan (LAM) is one of the three main glycolipids in MAGP complex. Fifteen percent of the dry weight of M. tuberculosis's wall comprises of LAM.(6) LAM is a potential antigen, which is able to mark the $M$. tuberculosis infection. LAM is not degraded in the human body (7), secreted by infected alveolar macrophages (8) and rising levels of this antigen is comparable with the increase of bacterial burden (9). LAM size resembles the size of myoglobin (17.5 kDa) causing this antigen in its unbound form can pass through the glomerular filtration easily on people with normal glomerular function so that LAM antigen can be detected from urine.(10)

The use of urine samples for M. tuberculosis antigens detection has several advantages. Urine sample can be obtained easily compare with serum and sputum samples. Urine can be obtained in large volumes without invasive procedure.(11) Urine collection and processing expose laboratory workers to lower health risk compare with serum and sputum samples.

The sensitivity of the LAM antigen examination in urine samples ranged between $2.4-93 \%$ with a specificity ranging from $87-99 \% .(12-15)$ The positivity of LAM examination in urine was higher in patients with human immunodeficiency virus (HIV) infection, particularly in patients with cluster of differentiation (CD) $4<200$ cells/ $\mu \mathrm{L}$. $(14,16)$ The wide range of sensitivity in the various studies may be influenced by the analytical sensitivity of the detection tool. Because of its potential in detecting mycobacterial infection, some researchers suggest to conduct researches regarding the methods to increase sensitivity of the LAM examination.(12-14) Sensitivity of antigen detection in body fluids can be improved by concentrating the fluid before performing immunoassay examination. Concentrating urine samples has been shown to increase the detection sensitivity of histoplasma capsulatum antigen $(17,18)$, Streptococcus pneumoniae $(19,20)$ and Legionella pneumophila $(21)$. It has been shown that the sensitivity of the enzyme-linked immunosorbent assay (ELISA) method for LAM in unconcentrated urine tuberculosis patients without HIV infection was 7\%.(22) The sensitivity was increased to $57 \%$ by concentrating the urine samples 100 times.(22) Population in this study consisted of patients with suspected pulmonary, extrapulmonary and latent tuberculosis.(22) Gold standard used in this study was smear staining with Ziehl-Neelsen method.(22) This study did not involve tuberculous meningitis patients.(22) To our knowledge, research on LAM antigen detection for diagnosis of tuberculosis infection in patients with tuberculous meningitis using concentrated urine has not been reported.

\section{Methods}

Subjects were patients who had been diagnosed with tuberculous meningitis according to Marais scoring system. Patients were hospitalized in Neurology Department of Dr. Hasan Sadikin Hospital, a tertiary province central hospital. Ethical approval was released by Medical Research Ethical Committee of Dr. Hasan Sadikin Hospital. The number of ethical clearance was LB 04.01/A05/EC/381/IX/2014. Examination materials used in this study were urine of tuberculous meningitis patients, all samples were taken in Dr. Hasan Sadikin Hospital. Determination of sample size in this study was based on a sample size formula for categorical descriptive study:

$$
\begin{aligned}
\mathrm{N}= & \frac{\mathrm{Z} \alpha \times \mathrm{p} \times(1-\mathrm{p})}{\mathrm{d}^{2}} \\
\mathrm{Z} \alpha & =1.96 \\
\mathrm{p} & =0.803 \\
\mathrm{~d} & =15 \%
\end{aligned}
$$

Hence, the number of samples needed for this study based on the calculation was 27 samples.

The suspected tuberculous meningitis patients with age of 18 years old or older were included. Subjects were categorized as possible, probable and definite. The categorization of possible and probable were made based on consensus score and computed tomography (CT) scan data availability. Patients were categorized as definite tuberculous meningitis if the cerebrospinal fluid culture indicate positive growth of $M$. tuberculosis (Table 1). Patients without CT scan examination data were categorized as possible 
tuberculous meningitis if the consensus score were 6-9 or as probable tuberculous meningitis if the consensus score were $\geq 10$. Patients with CT scan data were categorized as possible tuberculous meningitis if the consensus score were 6-11 or as probable tuberculous meningitis if the consensus score were $\geq 12$. This study was an observational study and cross sectionally designed.

Material used in this study is unconcentrated and concentrated urine from the middle stream portion of tuberculous meningitis patients. Patients or the closest relatives of the patients were given information regarding the procedure of the research. Written consent were taken if patients were agree to be involved in the research. Sterile urine tube and explanation regarding midstream urine collection were given. Urine sample from unconscious tuberculous meningitis patients were collected aseptically from sterile urine bag. All samples were morning voided urine. Samples were pooled and stored at $-20^{\circ} \mathrm{C}$ in less than 4 hours. At the day of analysis, urine samples were thawed at room temperature. To obtain concentrated urine, $5 \mathrm{~mL}$ urine samples were inserted into the sample room of the static concentrator. Concentration process lasts for 15-30 minutes and as much as $0.1 \mathrm{~mL}$ (100x concentrated) was produced. Cerebrospinal fluid cultured with Microscopic Observes Drug Susceptibility (MODS) were used as the gold standard procedure to classified the subject as definite tuberculous meningitis. The presence of LAM in unconcentrated and concentrated urine samples were detected with ELISA method. ELISA kit used in this study was manufactured by Cusabio Biotech Co. (Human Lipoarabinomannan ELISA Kit Lot.C0310041180; Catalog number CSB E09963h). This ELISA method employ a double sandwich assay principles. Monoclonal antibody was the capture antibody in this detection tool. Measurements were made at a wavelength of $450 \mathrm{~nm}$. The manufacturer of ELISA kit has determined that the cut off was obtained by adding a constant 0.10 to the optical density of the blank, the calculated cut off was 0.118 . Measurement result was categorized as positive if the optical density was higher than 0.118 and categorized as negative if the optical density was less than 0.118 . Sample processing and analysis were conducted at Clinical Pathology Department laboratory of Dr. Hasan Sadikin Hospital.

\section{Results}

Characteristics of research subjects were explained in Table 2. Most subjects were in the stage 2 of tuberculous meningitis. There were $33.3 \%$ subjects, classified as definite tuberculous meningitis. Only $14.9 \%$ subjects were diagnosed as tuberculous meningitis patients without evidence of tuberculosis on the site other than CNS.

The result of LAM detection in unconcentrated and concentrated urine of tuberculous meningitis patients with monoclonal antibody qualitative ELISA method can be seen in the Table 3. Patients with HIV positive were $29.6 \%$ of the subject studied. Positivity rate of urinary LAM among patients with HIV positive was $12.5 \%$. Positivity rate among patients categorized as definite tuberculous meningitis was $22.2 \%$. Positivity rate among patients categorized as probable was $11.1 \%$ and among possible tuberculous meningitis patients was $11.1 \%$. Research subjects with a positive HIV test results were $29.6 \%$ of all research subjects and dominated by male. Based on WHO clinical staging, research subjects with HIV in clinical stage 3 and 4 were $50 \%$ and $50 \%$. Positivity of urine LAM in stage 3 was $25 \%$ but no positivity were found in the group of clinical stage 4 .

\section{Discussion}

This study is the first study to examine LAM detection in unconcentrated and concentrated urine of tuberculous meningitis patients. Examination of LAM in unconcentrated and concentrated urine is expected to provide information

Table 1. Categorization of research subjects.

\begin{tabular}{lccc}
\hline Category & Consensus Score & $\begin{array}{c}\text { CT Scan Data } \\
\text { Availability }\end{array}$ & MODS \\
\hline Possible & $6-9$ & Not available & Negative \\
Probable & $2-11$ & Available & Not available \\
Definite & Regardless of consensus score and CT scan data & Positive \\
\hline MODS: Microscopic Observes Drug Susceptibility &
\end{tabular}

MODS: Microscopic Observes Drug Susceptibility 
Table 2. Characteristic of research subjects.

\begin{tabular}{lc}
\hline \multicolumn{1}{c}{ Characteristics } & n (\%) \\
\hline Age (year) & 33 \\
- Median & $19-64$ \\
- Range & \\
Gender & \\
- Male & $18(66.67 \%)$ \\
- Female & $9(33.33 \%)$ \\
& \\
Clinical stage of tuberculous \\
meningitis *
\end{tabular}

\section{Tuberculosis in the site other} than CNS

- Only pulmonary tuberculosis

$17(62.9 \%)$

- Disseminate tuberculosis

$6(22.2 \%)$

- Without tuberculosis on the site other than CNS

$4(14.9 \%)$

\section{HIV Infection}

- Positive

$8(29.6 \%)$

- Negative

$14(51.8 \%)$

- No data of HIV infection

$5(18.6 \%)$

* According to British Royal Medical Council

**According to Consensus Scoring system

Table 3. The result of LAM detection in unconcentrated and concentrated urine of tuberculous meningitis patients.

\begin{tabular}{ccc}
\hline \multirow{2}{*}{} & \multicolumn{2}{c}{$\begin{array}{c}\text { LAM Detection with } \\
\text { Qualitative ELISA }\end{array}$} \\
\cline { 2 - 3 } & LAM (+) & LAM (-) \\
\hline Unconcentrated Urine & 0 & $27(100 \%)$ \\
Concentrated Urine & $4(14.8 \%)$ & $23(85.2 \%)$ \\
\hline
\end{tabular}

about the role of concentrating urine sample to improve the performance of $\mathrm{M}$. tuberculosis antigen detection tool. The use of monoclonal antibody as capture antibody is expected to improve the specificity of the detection tool.

Research subjects in this study were in the age range of 18-64 years, this age range is not much different compare to previous studies conducted. $(22,24)$ Most of the research subjects were in clinical stage 2 of tuberculous meningitis. Most of the research subjects suffering from tuberculosis on the site other than CNS, especially in the lungs.

Low positivity of LAM detection in the urine among patients with pulmonary tuberculosis is consistent with previous research.(25) Urine of $22.7 \%$ patients with pulmonary tuberculosis were detected with LAM .(25) Low positivity results in urine LAM examination can be caused by immune condition of the research subjects as well as detection tool problems. LAM is an antigen that is secreted by $M$. tuberculosis and on it's way to environment passing through the structure of the wall and thus appears as a wallforming component.(26,27). M. tuberculosis-secreted LAM enters the circulation and is highly immunogenic causing humoral and cellular immune responses.(28) Humoral immune response in the formation of antibody against LAM leads to antibody-LAM complex. LAM in the bound form compare with unbound LAM, is more difficult to be detected by immunoassay. Impaired humoral immune responses increase the proportion of unbound LAM. LAM examination positivity in urine is determined by the proportion of unbound and bound LAM. Impaired cellular immune response leads to disruption of $M$. tuberculosis proliferation inhibition mechanism because cells that involved in cellular immune responses play a role in preventing the spread of M. tuberculosis.(29) Deficient of cellular immunity leads to the proliferation and spread of M. tuberculosis and LAM antigen, these account to the increase of bacterial load and antigen levels in the circulation in patients which in turn may lead to an increase in positivity of LAM detection in urine. Research subjects with a positive HIV test results were $29.6 \%$ of all research subjects and dominated by male. Based on WHO clinical staging, research subjects with HIV in clinical stage 3 and 4 were $50 \%$ and 50\%. Positivity in the group of subjects with HIV clinical stage 3 was $25 \%$ and no positivity was found in clinical stage 4 group. The higher positivity in clinical stage 3 group compared to clinical stage 4 group is contradictory with the lower immunity state of clinical stage 4 group.

Low positivity rate found in this study could be caused by the problem in the LAM ELISA kit detection limit. If the concentration of LAM in the sample studied was lower than the detection limit of the LAM ELISA kit, the examination will give negative result. The limit of detection is the lowest analyte concentration with the results of measurements that can be distinguished from blank.(30) Information regarding limit of detection was not provided by the manufacturer, thus the problem of detection limit in this study was recognized 
after researchers conducted a study to compare concentrated urine with 100x-diluted of concentrated urine. LAM examination of concentrated urine gave positive results, whereas LAM examination of 100x-diluted concentrated urine gave negative results. Results of these experiments indicated that the presence of LAM antigen in 100x diluted concentrated urine could not be detected by the ELISA kit. This experiment indicated that there was a certain detection limit of the ELISA kit. To investigate LAM detection limit of this ELISA kit may need further investigation.

This study used morning voided urine as sample, in order to obtain the most concentrated urine sample. Furthermore, the urine samples were concentrated with static concentrators. This efforts were expected to increase the levels of antigen per unit volume of urine. Savolainen studied the similar effort to concentrate urine samples 100x, this study showed that the efforts can increase the ELISA sensitivity in detecting LAM antigen from $7 \%$ to $57 \%$.(22) The results of Savolainen study could not be reproduced, since there are differences in the tools being used. This study used Cusabio ${ }^{\circledR}$ ELISA kit and static concentrators, previous study done by Savolainen used Clearview ${ }^{\circledR}$ ELISA kit and centrifugal concentrators.(22) Centrifugal concentrator utilizing centrifugation force. Centrifugal force can overcome the problem of the filter blockade that can be found in the static concentrators.(31) Centrifugator with larger bucket diameters is needed by centrifugal concentrators, this type of centrifugator is not available in most clinical laboratories. Static concentrators were chosen because this type of concentrator do not require installment of new and costly equipment. The difference in ELISA kit and concentrator used can be the cause of different results between this study and Savolainen study.

Reither experienced this problem when was trying to reproduce the results of Boehme study. Boehme's and Reither's studies used different ELISA kits, although from the same manufacturer. $(23,25)$ Reither analyzed the differences in the results of his research with the one of Boehme and came to conclusion that the manufacturer performed different producing methods for the ELISA kits. The most distinguishable difference of manufacturing method was in antibody coating method. Thus, Reither concluded that difference in ELISA kit manufacturing methods can cause differences in the results.(25) Savolainen used qualitative ELISA kit with polyclonal antibody as the capture antibody.(22) This study used qualitative ELISA kit with monoclonal antibody as the capture antibody. The use of monoclonal antibodies for the detection of antigens have the advantage of being able to increase the specificity of the examination and reduce the possibility of batch to batch variation which is commonly encountered in the use of polyclonal antibodies. The use of monoclonal antibody to increase specificity at the expense of sensitivity may be lower, thus lowering the positivity rate.

\section{Conclusion}

The effort to concentrate urine sample of tuberculous meningitis patients produce a low increment of positivity rate as much as $14.8 \%$ thus detection of mycobacterial LAM with qualitative monoclonal ELISA is insignificantly improved with this effort.

Further study is needed to quantify the difference in diagnostic advantage between the usage of static and centrifugal concentrators for urine samples. Detection of LAM from urine samples for screening purposes might be better done with polyclonal antibody coated ELISA kit compared to monoclonal antibody coated ELISA kit, however further studies is also required to back up this statement.

\section{References}

1. Eurosurveillance editorial team WHO. Global tuberculosis report 2013. 18th ed. Floyd K, Raviglione M, Baddeley A, Dean A, Dias HM, Falzon D, et al., editors. Geneva: WHO Press; 2013.

2. Vitoria M, Granich R, Gilks CF, Gunneberg C, Hosseini M, Were W, et al. The global fight against HIV/AIDS, tuberculosis, and malaria: current status and future perspectives. Am J Clin Pathol. 2009; 131: 844-8.

3. Grange JM, Zumla A. The global emergency of tuberculosis: what is the cause? The journal of the Royal Society for the Promotion of Health. 2002; 122: 78-81.

4. Raviglione MC, O'Brien RJ. Tuberculosis. In: Fauci AS, Dennis L, Kasper M, Longo DL, Braunwald E, Hauser SL, et al., editors. Harrison's Principles of Internal Medicine. 17th ed. New York: McGraw-Hill; 2008.

5. Marais S, Thwaites G, Schoeman JF, Torok ME, Misra UK, Prasad K, et al. Tuberculous meningitis: a uniform case definition for use in clinical research. Lancet Infect Dis. 2010; 10: 803-12.

6. Karakousis PC, Bishai WR, Dorman SE. Mycobacterium tuberculosis cell envelope lipids and the host immune response. Cell Microbiol 2004; 6: 105-16.

7. Hunter SW, Gaylord H, Brennan PJ. Structure and antigenicity of the phosphorylated lipopolysaccharide antigens from the leprosy and tubercle bacilli. J Biol Chem. 1986; 261: 12345-51.

8. Beattya WL, Rhoadesa ER, Ullricha HJ, Chatterjeec D, Heuserb JE, Russell DG. Trafficking and release of mycobacterial lipids. Traffic. 2000; $1: 235-47$

9. Minion J, Leung E, Talbot E, Dheda K, Pai M, Menzies D. Diagnosing tuberculosis with urine lipoarabinomannan: systematic review and meta-analysis. Eur Respir J. 2011; 38: 1398-405. 
10. Wood R, Racow K, Lawn SD, Bekker LG, Middelkoop K, Vogt M, et al. Lipoarabinomannan in urine during tuberculosis treatment: association with host and pathogen factors and mycobacteriuria. BMC Infect Dis. 2012; 12: 1-11.

11. Tuuminen T. Urine as a specimen to diagnose infections in twentyfirst century: focus on analytical accuracy. Front Immunol. 2012; 3: $1-6$.

12. Peter J, Green C, Hoelscher M, Mwaba P, Zumla A, Dheda K. Urine for the diagnosis of tuberculosis: current approaches, clinical applicability, and new developments. Curr Opin Pulm Med. 2010; 16: $262-70$.

13. Sarkar P, Biswas D, Sindhwani G, Rawat J, Kotwal A, Kakati B. Application of lipoarabinomannan antigen in tuberculosis diagnostics: current evidence. Postgrad Med J. 2014; 90: 155-63.

14. Lawn SD, Kerkhoff AD, Vogt M, Wood R. Diagnostic accuracy of a low-cost, urine antigen, point-of-care screening assay for HIVassociated pulmonary tuberculosis before antiretroviral therapy: a descriptive study. Lancet Infect Dis. 2012; 12: 201-9.

15. Rustandi D. Gambaran pemeriksaan antigen lipoarabinomannan metode enzyme linked immunosorbent assay pada urin anak penderita tuberkulosis [Thesis]. Bandung: Universitas Padjajaran; 2011.

16. Dheda K, Davids V, Lenders L, Roberts T, Meldau R, Ling D, et al. Clinical utility of a commercial LAM-ELISA assay for TB diagnosis in HIV-infected patients using urine and sputum samples. PloS One. 2010; 5: e9848.

17. Wheat LJ, Kohler RB, Tewari RP. Diagnosis of disseminated histoplasmosis by detection of histoplasma capsulatum antigen in serum and urine specimens. N Engl J Med. 1986; 314: 83-8.

18. Srinivasan A, Kleiman MB, Debelenko L, Stokes DC, De Vincenzo J, Wheat JL. False-negative histoplasma antigen in acute pulmonary histoplasmosis: the value of urinary concentration by ultrafiltration and heat denaturation of serum proteins in detection of histoplasma antigen. Pediatr Infect Dis J. 2009; 28: 447-9.

19. Dominguez J, Blanco S, Rodrigo C, Azuara M, Gali N, Mainou $A$, et al. Usefulness of urinary antigen detection by an immunochromatographic test for diagnosis of pneumococcal pneumonia in children. J Clin Microbiol. 2003; 41: 2161-3.

20. Marcos MA, Jimenez de Anta MT, de la Bellacasa JP, Gonzalez J, Martinez E, Garcia E, et al. Rapid urinary antigen test for diagnosis of pneumococcal community-acquired pneumonia in adults. Eur
Respir J. 2003; 21: 209-14.

21. Guerrero C, Toldos CM, Yague G, Ramirez C, Rodriguez T, Segovia M. Comparison of diagnostic sensitivities of three assays (Bartels enzyme immunoassay [EIA], Biotest EIA, and Binax NOW immunochromatographic test) for detection of Legionella pneumophila serogroup 1 antigen in urine. J Clin Microbiol. 2004; 42: 467-8

22. Savolainen L, Kantele A, Sandboge B, Siren M, Valleala H, Tuompo $\mathrm{R}$, et al. Modification of clearview tuberculosis (TB) enzyme-linked immunosorbent assay for TB patients not infected with HIV. Clin Vaccine Immunol. 2013; 20: 1479-82.

23. Boehme C, Molokova E, Minja F, Geis S, Loscher T, Maboko L, et al. Detection of mycobacterial lipoarabinomannan with an antigencapture ELISA in unprocessed urine of Tanzanian patients with suspected tuberculosis. Trans R Soc Trop Med Hyg. 2005; 99: 893900 .

24. Ganiem AR, Parwati I, Wisaksana R, van der Zanden A, van de Beek $\mathrm{D}$, Sturm P, et al. The effect of HIV infection on adult meningitis in Indonesia: a prospective cohort study. AIDS. 2009; 23: 2309-16.

25. Reither K, Saathoff E, Jung J, Minja LT, Kroidl I, Saad E, et al. Low sensitivity of a urine LAM-ELISA in the diagnosis of pulmonary tuberculosis. BMC Infect Dis. 2009; 9: 141.

26. Lemassu A, Daffe M. Structural features of the exocellular polysaccharides of Mycobacterium tuberculosis. Biochem J. 1994; 297 (Pt 2): 351-7.

27. Chatterjee D, Lowell K, Rivoire B, McNeil MR, Brennan PJ. Lipoarabinomannan of mycobacterium tuberculosis. J Biol Chem. 1992; 267: 6234-9.

28. Briken V, Porcelli SA, Besra GS, Kremer L. Mycobacterial lipoarabinomannan and related lipoglycans: from biogenesis to modulation of the immune response. Mol Microbiol. 2004; 53: 391403.

29. Strohmeier GR, Fenton MJ. Roles of lipoarabinomannan in the pathogenesis of tuberculosis. Microbes Infect. 1999; 1: 709-17.

30. Armbruster DA, Pry T. Limit of blank, limit of detection and limit of quantitation. Clin Biochem Rev. 2008; 29 (Suppl 1): S49-52.

31. Christenson RH, Studenberg SD, Beck-Davis S, Sedor FA. Digoxinlike immunoreactivity eliminated from serum by centrifugal ultrafiltration before fluorescence polarization immunoassay of digoxin. Clin Chem. 1987; 33: 606-8. 